\title{
Investigation of Discordant SARS-CoV-2 RT-PCR Results Using Neat Saliva
}

\author{
Dawn White \\ McMaster University \\ Jimmy Gu \\ McMaster University \\ Catherine-Jean Steinberg \\ McMaster University \\ Deborah Yamamura \\ McMaster University \\ Bruno J. Salena \\ McMaster University \\ Cynthia Balion \\ McMaster University \\ Alfredo Capretta \\ McMaster University \\ Yingfu Li \\ McMaster University \\ John D. Brennan ( $\nabla$ brennanj@mcmaster.ca ) \\ McMaster University
}

\section{Research Article}

Keywords: RT-PCR, COVID-19

Posted Date: October 15th, 2021

DOl: https://doi.org/10.21203/rs.3.rs-948759/v1

License: @ (i) This work is licensed under a Creative Commons Attribution 4.0 International License.

Read Full License 


\section{Abstract}

Saliva is an attractive sample for coronavirus disease 2019 testing due its ease of collection and amenability to detect viral RNA without processing. Using a direct-to-RT-PCR method with saliva selfcollected from confirmed COVID-19 positive volunteers, we observed $32 \%$ false negative results. Confirmed negative and healthy volunteer samples spiked with $10^{6}$ genome copies $/ \mathrm{mL}$ of heatinactivated severe acute respiratory syndrome coronavirus 2 showed false negative results of $10 \%$ and $13 \%$, respectively. Additional sample heating or dilution of the false negative samples conferred modest improvement. These results highlight the potential to significantly underdiagnose COVID-19 infections when testing directly from heterogeneous neat saliva samples.

\section{Introduction}

Severe acute respiratory syndrome coronavirus 2 (SARS-CoV-2) has impacted daily life on a global scale since its emergence in December 2019. Scientists rallied quickly to gather and disseminate information to create sensitive molecular detection methods for SARS-CoV-2 to protect the population ${ }^{1}$. Public health authorities have relied heavily on the "gold standard" reverse-transcription polymerase chain reaction (RTPCR) testing of nasopharyngeal swabs (NPS). However, this type of sample collection requires professional healthcare workers for procurement, which has prompted researchers to evaluate saliva as a simple, self-collectable specimen. Many studies have shown comparable diagnostic sensitivity for viral genomic RNA extracted from saliva specimens versus NPS when using RT-PCR for COVID-19 detection². To further increase simplicity, several methods, such as the covidSHIELD test developed at the University of Illinois Urbana-Champaign (UIUC) ${ }^{3}$, the SalivaDirect test developed at Yale ${ }^{4}$, and the SalivaSTAT method developed at the Medical College of Georgia ${ }^{5}$, utilize direct analysis from unprocessed saliva to avoid the RNA extraction step.

Our groups are actively involved in the development of a point-of-care antigen test for COVID-19 utilizing neat saliva as the specimen. We chose the covidSHIELD RT-PCR processing method to aid in validation of our test ${ }^{6}$ due to its relative ease and reported sensitivity. However, it was observed during test development that the inherent complexity of neat saliva ${ }^{7}$ produced a significant number of false negative SARS-CoV-2 RT-PCR results, prompting us to investigate further.

\section{Methods}

\section{Assessment center and healthy volunteer saliva samples}

The research and all associated experimental protocols, including those related to sample collection, processing and testing, were approved by the Research Ethics Board of McMaster University and its affiliated hospitals (Hamilton Health Sciences and St. Joseph's Healthcare). The research was performed in accordance with the institutional guidelines/regulations and the Declaration of Helsinki. Informed consent was obtained from all participants ( $\geq 18$ years of age) and/or their legal guardians attending a 
Hamilton Health Sciences affiliated COVID-19 assessment center (Hamilton, Ontario) for NPS SARS-CoV2 testing. Saliva ( 1 to $5 \mathrm{~mL}$ ) was self-collected under supervision following the instructions accompanying a provided saliva collection kit immediately after the nasopharyngeal swab. Patients were confirmed as SARS-CoV-2 positive (22 patients: NPS positive) or negative (92 patients: NPS negative) from the NPS sample at the Hamilton Regional Laboratory Medicine Program at St. Joseph's Healthcare (Hamilton) using an established method ${ }^{8}$. Patient samples were stored at $4^{\circ} \mathrm{C}$ for $<72$ hours prior to transport to McMaster University for storage at $-80^{\circ} \mathrm{C}$. Healthy adult volunteers (23) from the McMaster University research community provided saliva samples in sterile $50 \mathrm{~mL}$ polypropylene tubes following the same self-collection instructions. Samples were aliquoted then frozen or used on the same day as collection.

\section{RT-PCR}

RT-PCR was performed using the Power SYBR $\circledast$ Green RNA-to- $\mathrm{C}_{T}{ }^{\mathrm{T}}{ }^{\mathrm{T}}$ 1-step Kit (Fisher Scientific, Ottawa, Ontario, Canada) with the CDC N1 and RNaseP forward and reverse primers from Integrated DNA Technologies (IDT: NC, USA). All assessment center and healthy volunteer saliva samples were processed based on the original covidSHIELD method described by Ranoa et al. ${ }^{3}$. Briefly, a 20-50 $\mu \mathrm{L}$ aliquot of the saliva sample was heated at $95^{\circ} \mathrm{C}$ for 30 minutes followed by the addition of an equal volume of $2 x$ TBETw buffer (100 mM Tris pH 7.5, 90 mM boric acid, 1 mM EDTA, 1\% Tween-20). Saliva samples were then incubated at room temperature for 5 minutes to allow any natural salivary particulates to settle to the bottom of the tube. The sample supernatant $(5 \mu \mathrm{L})$ was mixed with $15 \mu \mathrm{L}$ of RT-PCR master mix (prepared as instructed) containing $200 \mathrm{nM}$ of $\mathrm{N} 1$ or RNaseP forward and reverse primers; samples were run on a Biorad CFX96 Touch Real Time PCR system (Hercules, CA, USA). The cycling parameters were: RT $-48^{\circ} \mathrm{C}, 30$ minutes; $\mathrm{PCR}-95^{\circ} \mathrm{C}, 10$ minutes, followed by 40 cycles of $95^{\circ} \mathrm{C}$ for 15 seconds then $60^{\circ} \mathrm{C}$ for 1 minute; melt curve $-95^{\circ} \mathrm{C}$ for 15 seconds, $60^{\circ} \mathrm{C}$ for 15 seconds, $95^{\circ} \mathrm{C}$ for 15 seconds. Based on standard curves (Supplementary Figure S1) and the limit of detection obtained with these reaction conditions (see details in Supplementary), a threshold cycle value $\left(C_{t}\right.$ value $)$ of $>36$ was considered negative.

\section{Spiking saliva samples with heat-inactivated SARS-CoV-2; heating and diluting saliva samples}

Heat-inactivated 2019 Novel Coronavirus (Isolate USA-WA/2020) was purchased from the ATCC through Cedarlane (Burlington, Ontario, Canada). SARS-CoV-2 NPS negative and healthy volunteer saliva samples were spiked with $4.2 \times 10^{6}$ genome copies $(\mathrm{gc}) / \mathrm{mL}$ of heat-inactivated SARS-CoV-2 prior to processing for RT-PCR as described above. Saliva samples that were heated prior to spiking and RT-PCR processing were incubated at $95^{\circ} \mathrm{C}$ for 30 minutes. Saliva samples that were diluted prior to RT-PCR processing were done so with an equal volume of sterile RNase-free water.

\section{Results}

All saliva samples included in this study gave a positive result for the human RNaseP internal control using the processing method of the covidSHIELD RT-PCR laboratory developed test ${ }^{3}$ (Supplementary 
Table S1).

To test our workflow and reagents with the covidSHIELD RT-PCR processing method, we initially examined SARS-CoV-2 negative saliva samples (NPS negative) spiked with $10^{6} \mathrm{gc} / \mathrm{mL}$ heat-inactivated SARS-CoV-2. At this "viral load", a $C_{t}$ value of 29 was expected based on our calibration data (Supplementary Figure S1). We observed great RT-PCR negativity $\left(C_{t}\right.$ value $\left.>36\right)$ with the spiked samples when the processed saliva was mixed prior to addition to the amplification mixture (Figure 1a). Allowing the same samples to settle improved detection markedly, therefore this settling step was included in all subsequent experiments.

All assessment center saliva samples that tested SARS-CoV-2 negative by NPS also tested negative using the RT-PCR method (100\% specificity). However, only 15 of the 22 SARS-CoV-2 positive NPS samples showed a positive result using the RT-PCR method with the paired saliva sample ( $68 \%$ sensitivity, see Figure 1b: NPS positive) with an average $C_{t}$ value of $31.7 \pm 2.5$ and $C_{t}$ range of $26.6-34.3$ (Supplementary Figure S2).

This observation led us to question the effect of saliva sample quality (viscosity, particulates) on the test. Physical examination of all the study samples revealed varying viscosity (ranging from water-like to highly viscous with a heterogenous composition), clarity (clear, cloudy, turbid) and degree of particulate matter ( $<10 \%$ to $>75 \%$ of the sample volume). We evaluated 50 representative SARS-CoV-2 negative saliva samples-from clear and watery to turbid and viscous-by spiking each with $10^{6} \mathrm{gc} / \mathrm{mL}$ heatinactivated SARS-CoV-2. Despite having identical "viral loads", these samples showed significant variability in the $C_{t}$ values (average $C_{t}$ value of $31.0 \pm 2.2 ; C_{t}$ range of $27.4-34.9$ ); more concerning was that five samples showed a negative RT-PCR result (Figure 1b: NPS negative; Supplementary Figure S2) that did not correlate with the physical quality of the sample, suggesting the presence of chemical components that interfered with the assay.

We then questioned whether saliva samples collected from assessment center volunteers, who presented with symptoms of SARS-CoV-2 infection or suspected exposure to the virus, caused greater interference than samples from healthy people. A set of 23 healthy volunteer saliva samples were spiked and analyzed as described above. A similar result was obtained wherein three of the samples produced a negative RT-PCR result even after spiking (Figure 1b: Healthy volunteer; Supplementary Figure S2) (average $C_{t}$ value of $31.5 \pm 1.7 ; C_{t}$ range of $28.5-34.1$ ).

Saliva is a complex matrix reported to contain unknown RT-PCR inhibitors ${ }^{9}$, and RNAses that could degrade viral RNA upon release from the capsid ${ }^{10}$. To assess if such inhibitors contributed to the observed false negative results with the spiked saliva samples, four SARS-CoV-2 negative and three healthy volunteer saliva samples that did not respond to spiking were heated at $95^{\circ} \mathrm{C}$ for 30 minutes to denature/degrade salivary interferants prior to spiking and processing as before. Four of the seven samples showed positive $C_{t}$ values (average $\Delta C_{t}$ [spiked-heated] of $7.9 \pm 1.7 ; C_{t}$ range of $28.6-32.9$ ) (Figure 2a). 
To determine if chemical interferants could be sufficiently reduced by dilution, which is often used to reduce viscosity ${ }^{11}$ and aid with automated sample processing ${ }^{12}$, saliva samples were diluted $50 \%$ before the $50 \%$ dilution step incorporated in the RT-PCR processing method creating a $25 \%$ saliva solution prior to addition to the RT-PCR reaction. The saliva samples chosen for examination were four SARS-CoV-2 positive samples that tested negative using the RT-PCR method and two healthy volunteer samples whose saliva remained negative after spiking with SARS-CoV-2. The SARS-CoV-2 positive samples remained negative after dilution while the two healthy volunteer samples gave positive results, with a reduction in $C_{t}$ value of 7.6 and 6.7 units (Figure $2 b$ ).

\section{Discussion}

Accurate reporting of COVID-19 infections to monitor populations and control outbreaks has been a primary goal worldwide from the start of the pandemic. Among the various methods currently in use, tests using saliva have the advantage of easy sample acquisition, which could allow collection without a health-care practitioner. However, our study has indicated that neat saliva poses a problem for conventional RT-PCR assays due to complications surrounding its physical attributes (viscosity, heterogeneity) and unidentified chemical constituents.

A key observation was that dispersal of saliva particulates via sample mixing prior to the RT-PCR assay increased the number of observed false negative results and increased $C_{t}$ values generally, even in positive samples (Figure 1a). Allowing the prepared sample to settle for at least 5 minutes after processing greatly improved RT-PCR detectability. In our hands, brief centrifugation worked as well as settling (Supplementary Figure S3), although Ranoa et al. ${ }^{3}$ did not observe the same benefit. Based on the visual assessment of several hundred assessment center negative saliva samples including and beyond those used for this study, there was no obvious correlation between any physical attribute and an erroneous RT-PCR result. Furthermore, the rate of false negative results did not seem to depend on the sample group (NPS positive, NPS negative, Healthy volunteer) (Figure 1b; Supplementary Figure S2).

Pre-heating of seven saliva samples that consistently returned false negative results after spiking with heat-inactivated virus showed marked improvement-four samples returned a positive result (Figure 2a, black triangles)-suggesting that some inhibitory components in the saliva could be disrupted or degraded. However, the remaining three samples showed resistance to the treatment, and it was unclear why the long heating step already included in the covidSHIELD method was not sufficient for inactivation of the interferants. Sample dilution was unable to produce correct results for false negative saliva samples (NPS positive, saliva RT-PCR negative) (Figure 2b, grey circle and square), though this method did remedy false negative results from spiked healthy samples.

While the utility of saliva as a diagnostic specimen cannot be disputed ${ }^{13}$, and even considering the documented reduction in detection sensitivity relative to NPS samples ${ }^{14}$, our observations provide evidence for a potentially significant issue with a high false negative rate when using unprocessed saliva samples for COVID-19 RT-PCR testing. Indeed, the original manuscript describing the covidSHIELD 
method $^{3}$ reported $11 \%$ false negative results upon initial testing, but $100 \%$ sensitivity upon retesting; Kandel et al. ${ }^{15}$ reported 38 of 432 samples (8.8\%) as invalid upon initial testing, improving to three invalid samples after retesting; and Sahajpal et al. ${ }^{5}$ reported $5 \%$ invalid results (though no false negatives).

Our study indicated that valid samples showing expected $C_{t}$ values for the control RNaseP sequence could repeatedly result in a false negative SARS-CoV-2 result, suggesting an inherent issue with interferants in some saliva samples. We note that several other factors could lead to variability in saliva samples, such as the timing of sample collection with respect to disease progression, symptoms, and time of day; and the volume of saliva provided. We also recognize that disease prevalence in the population at the time of sample collection and our limited number of samples may have contributed to our observations. However, general agreement in the rate of false negative SARS-CoV-2 RT-PCR results across 137 samples from assessment centre attendees and healthy volunteers presented here, and seen in other studies, points to unknown factors that produce significant false negative results with a widely used RT-PCR method. These results indicate that while direct use of unprocessed saliva is desirable to increase the simplicity and reduce the cost for COVID-19 testing, the relatively high rate of false negative results may require additional sample processing, such as RNA extraction, to reduce the possibility of COVID-19 positive citizens unknowingly transmitting the virus within the community.

\section{Declarations}

Author contributions. DW, AC, YL and JDB designed the experiments. DW performed the experiments; DW, $\mathrm{CB}, \mathrm{AC}, \mathrm{YL}$ and JDB analyzed the data. JG, CJS, DY and BJS oversaw patient saliva collection. DW and JDB wrote the manuscript; DW, DY, CB, AC, YL and JDB edited the manuscript.

Financial support. This work was supported by the Canadian Institutes for Health [grant number 446998] and the McMaster University COVID-19 Research Fund. We also thank Maryella Leggatt for providing financial support of this work through a generous donation. JDB holds the Canada Research Chair in Point-of-Care Diagnostics.

Competing interests. All authors declare no completing interests.

\section{References}

1. Pérez-López, B. \& Mir, M. Commercialized diagnostic technologies to combat SARS-CoV-2: Advantages and disadvantages. Talanta.225,121898(2021).

2. Yu, C. Y., Chan, K. G., Yean, C. Y. \& Ang, G. Y. Nucleic acid-based diagnostic tests for the detection SARS-CoV-2: An update.Diagnostics.11, 53(2021).

3. Ranoa, D. R. E. et al. Saliva-based molecular testing for SARS-CoV-2 that bypasses RNA extraction. Preprint at https://doi, https://doi.org/org/10.1101/2020.06.18.159434 (2020).

4. Vogels, C. B. F. et al. SalivaDirect: simple and sensitive molecular diagnostic test for SARS-CoV-2 surveillance. Preprint at https://doi, https://doi.org/org/10.1101/2020.08.03.20167791 (2020). 
5. Sahajpal, N. S. et al. SalivaSTAT: Direct-PCR and pooling of saliva samples collected in healthcare and community setting for SARS-CoV-2 mass surveillance.Diagnostics.11,904(2021).

6. Zhang, Z. et al. High affinity dimeric aptamers enable rapid electrochemical detection of wild-type and B.1.1.7 SARS-CoV-2 in unprocessed saliva. Angew. Chem. Int. Ed. Online at https://doi.org/10.1002/anie.202110819 (2021).

7. Han, P. \& Ivanovski, S. Saliva - friend and foe in the COVID-10 outbreak.Diagnostics.10,290(2020).

8. LeBlanc, J. J. et al. Real-time PCR-based SARS-CoV-2 detection in Canadian laboratories.J. Clin. Virol.128,104433(2020).

9. Ochert, A. S., Boulter, A. W., Birnbaum, W., Johnson, N. W. \& Teo, C. G. Inhibitory effect of salivary fluids on PCR: potency and removal.Genome Res.3,365-368(1994).

10. Nishibata, Y. et al. RNase in the saliva can affect the detection of severe acute respiratory syndrome coronavirus 2 by real-time one-step polymerase chain reaction using saliva samples. Pathol. Res. and Prac.220,153381(2021).

11. Alkhateeb, K. J., Cahill, M. N., Ross, A. S., Arnold, F. W. \& Snyder, J. W. The reliability of saliva for the detection of SAR-CoV-2 in symptomatic and asymptomatic patients: insights on the diagnostic performance and utility for COVID-19 screening.Diagn. Microbiol. Infect. Dis.101,115450(2021).

12. Landry, M. L., Criscuolo, J. \& Peaper, D. R. Challenges in use of saliva for detection of SARS- CoV-2 in symptomatic outpatients.J. Clin. Virol.130,104567(2020).

13. Butler-Laporte, G. et al. Comparison of saliva and nasopharyngeal swab nucleic acid amplification testing for detection of SARS-CoV-2: a systematic review and meta-analysis. JAMA Intern. Med. 181(3),353-360(2021).

14. Jamal, A. J. et al. Sensitivity of nasopharyngeal swabs and saliva for the detection of severe acute respiratory syndrome coronavirus 2.Clin. Infect. Dis.72(6),1064-1066(2021).

15. Kandel, C. et al. Detection of SARS-CoV-2 from saliva as compared to nasopharyngeal swabs in outpatients. Viruses.12,1314(2020).

\section{Figures}




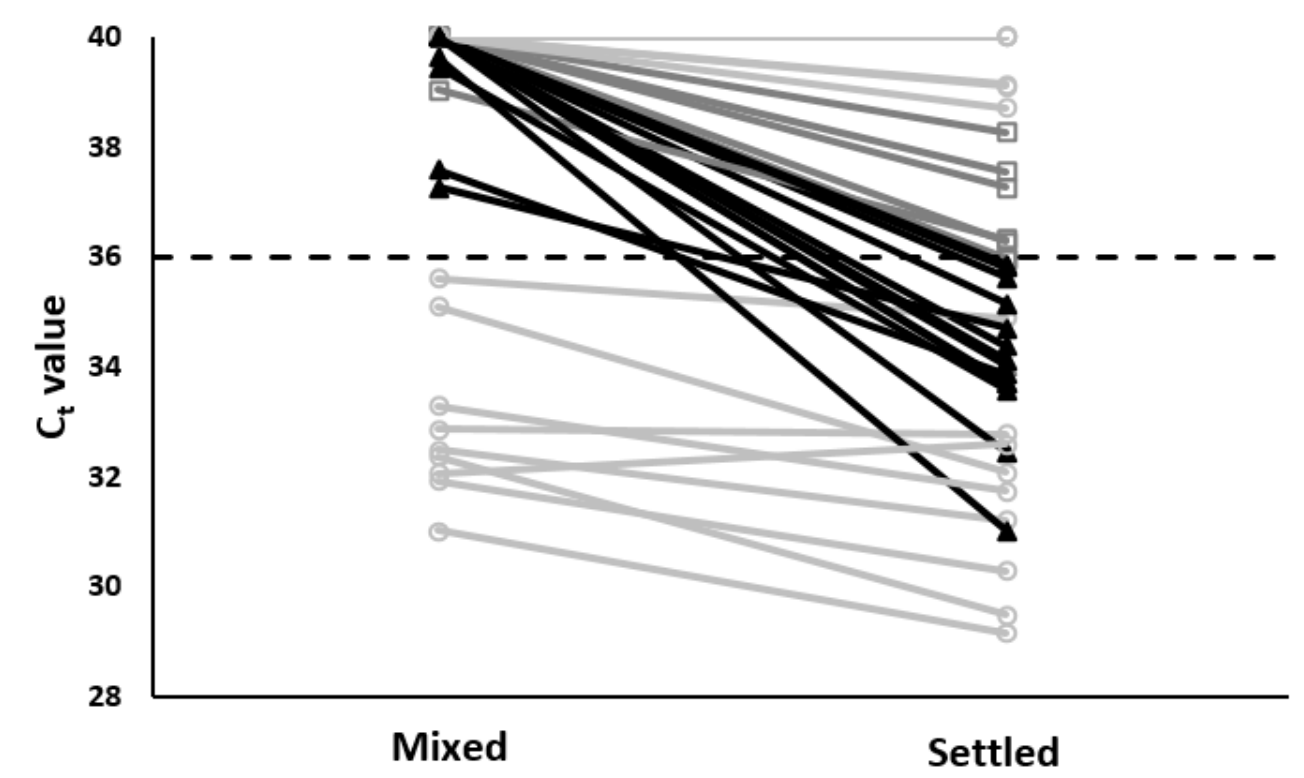

b

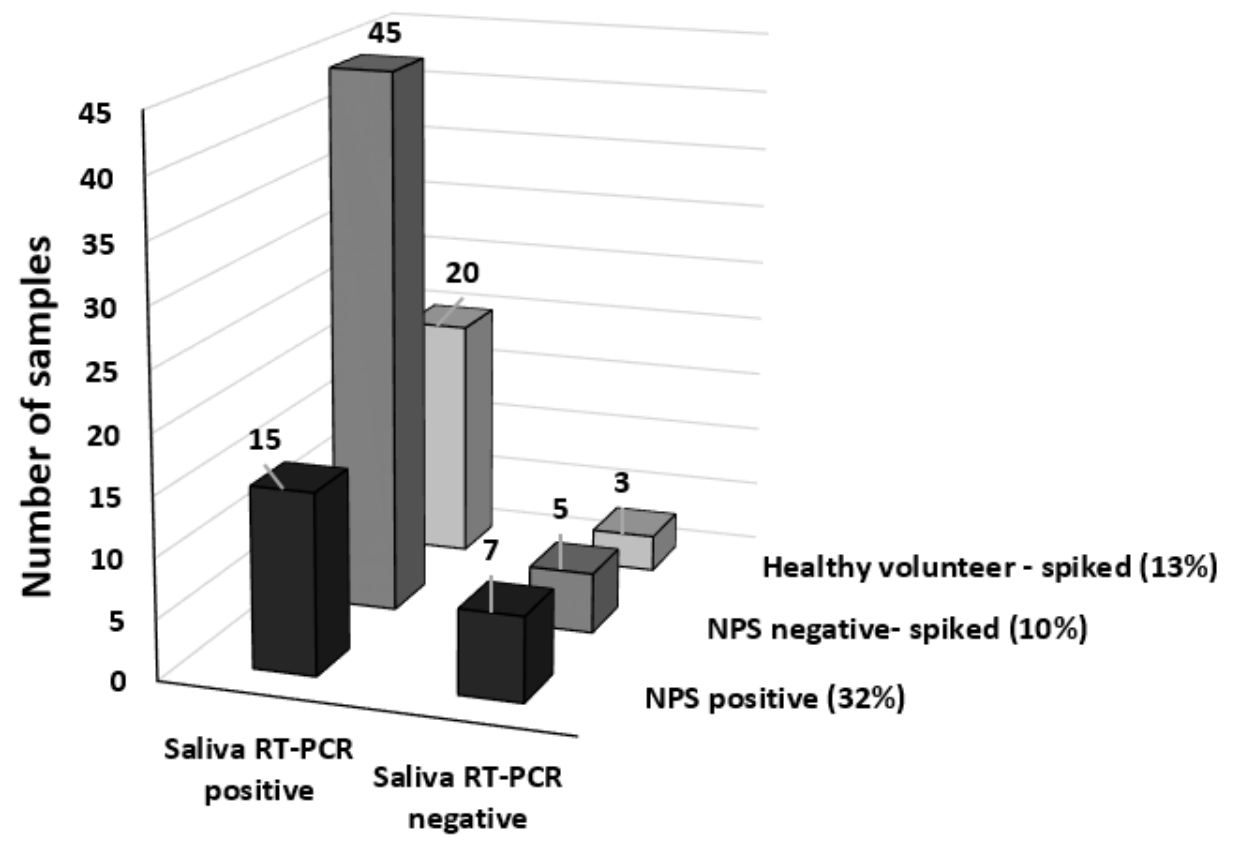

\section{Figure 1}

Detection of SARS-CoV-2 in unprocessed saliva samples from COVID-19 positive and negative volunteers. (a) Improved detection of spiked saliva samples allowed to settle after processing versus being mixed after processing. Solid black triangles represent samples that became detectable $(\mathrm{Ct}<36)$ after settling; dark grey open squares represent samples that showed improved Ct values but remained above the cutoff $\mathrm{Ct}$ value (Ct > 36, black dashed line); light grey open circles represent samples that were either 
detectable in both groups or did not show an appreciable change in Ct value. A Ct value of 40 represents a negative result. (b) Prevalence of false negative SARS-CoV-2 RT-PCR outcomes using neat saliva samples. The sample number is indicated above the data columns. The percent of false negatives is shown in brackets beside each group. All NPS positive saliva samples were tested as is. All NPS negative and healthy volunteer saliva samples were spiked with 106 genome copies/mL heat-inactivated SARSCoV-2 prior to testing. All samples tested at least in triplicate. See Supplementary Figure S2 for individual sample Ct values.

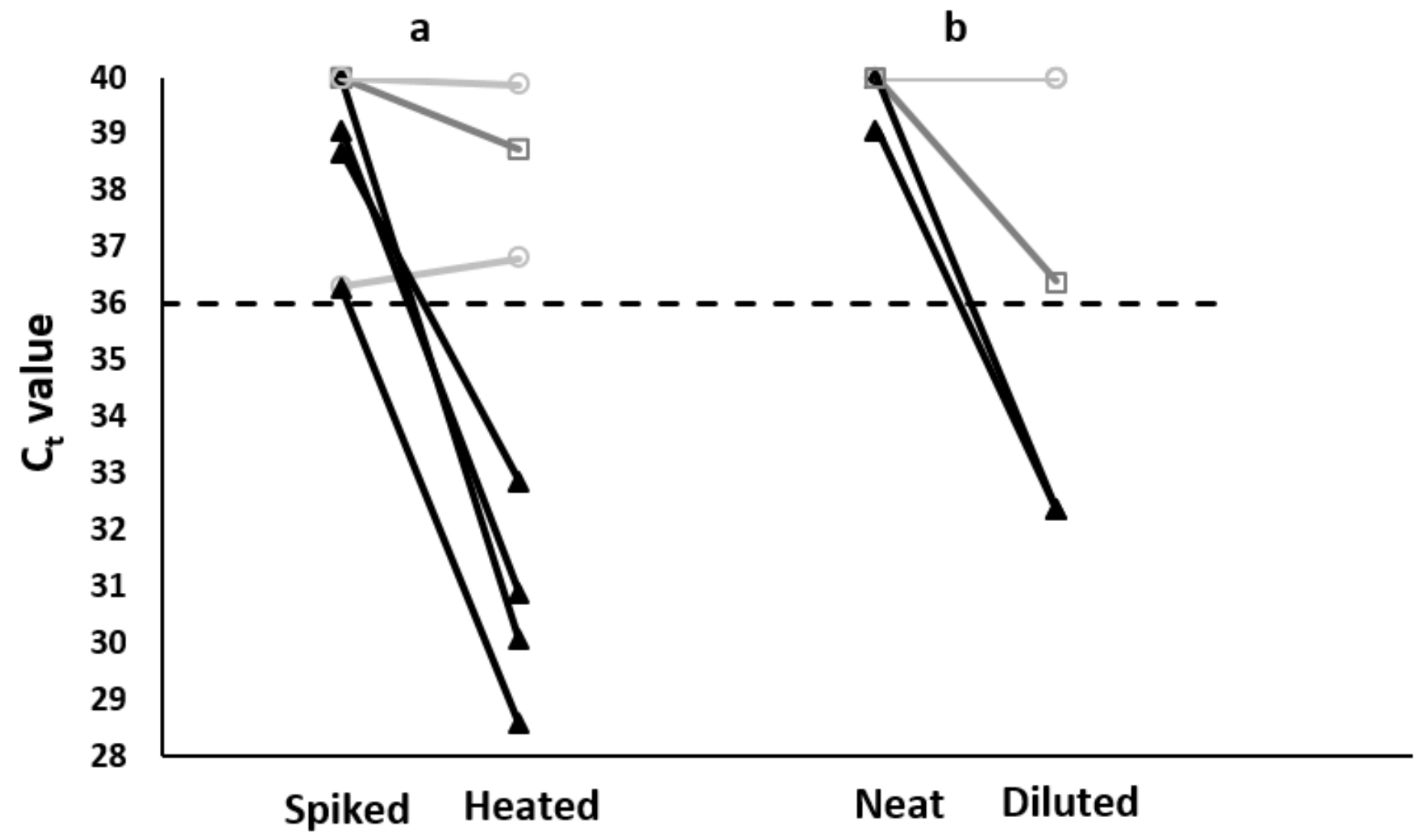

Figure 2

Effect of heat treatment or dilution of spiked saliva samples prior to testing. (a) Saliva samples from NPS negative or healthy volunteers that produced a false negative result after spiking with 106 genome copies/mL heat-inactivated SARS-CoV-2 (Spiked) compared with the same saliva samples heated prior to spiking (Heated). (b) Spiked saliva samples that produced a false negative result (Neat) tested after dilution (Diluted). Solid black triangles represent samples that became detectable $(\mathrm{Ct}<36)$ after settling; dark grey open squares represent samples that showed improved $\mathrm{Ct}$ values but remained above the cutoff $\mathrm{Ct}$ value ( $\mathrm{Ct}>36$, black dashed line); light grey open circles represent samples that were either detectable in both groups or did not show an appreciable change in Ct value (In panel B only, the light grey open circle represents 3 identical, undetectable samples.) A Ct value of 40 represents a negative result; the black dashed line at $\mathrm{Ct}=36$ represents the cut-off value for our system. Samples tested at least in duplicate. 


\section{Supplementary Files}

This is a list of supplementary files associated with this preprint. Click to download.

- SRSalivaPCRSupplementaryDatav1.docx 